\title{
20 Jahre im Dienst der Qualität in der ambulanten Medizin
}

\author{
Paula Bezzola ${ }^{a}$, Felix Huber ${ }^{b}$, Ruth Humbel ${ }^{c}$ \\ ${ }^{a}$ MPH, Geschäftsführerin EQUAM Stiftung; ${ }^{b}$ Dr. med., Präsident mediX Schweiz, Vizepräsident des Stiftungsrates EQUAM; \\ c Nationalrätin, Präsidentin des Stiftungsrats EQUAM
}

Ende Oktober feierte die EQUAM Stiftung ihr 20-Jahr-Jubiläum. Namhafte Redner lobten die Pionierarbeit der Stiftung für Qualität in der ambulanten Versorgung. Die Sprecher erwarten, dass die verabschiedete KVG-Revision zur Stärkung von Qualität und Wirtschaftlichkeit, welche Verbindlichkeit bei der Umsetzung von Qualitätsmassnahmen vorschreibt, die Relevanz der Arbeit weiter verstärken wird.

\section{Artikel 58 des KVG - die Geburtsstunde der EQUAM Stiftung}

Die Qualität im Gesundheitswesen wurde mit dem Krankenversicherungsgesetz (KVG) von 1996 zum ersten Mal ausdrücklich im Gesetz verankert (Art. 58: Qualitätssicherung). Der Gesetzgeber hat es der Ärzteschaft und den Versicherern überlassen, die Qualitätsverfahren bilateral festzulegen. Ärztegruppen, welche Budgetverträge mit Partnerversicherungen abgeschlossen hatten, sahen sich mit dem Verdacht der Billigmedizin konfrontiert. Daraus entstand das Bedürfnis nach einem Qualitätsnachweis durch eine externe, neutrale Instanz.

Drei leitende Ärzte aus HM-Organisationen - Dr. med. Kurt Hess, SanaCare, Dr. med. Felix Huber, mediX Schweiz, und Dr. med. Thomas Grüninger, Gesundheitsplan HMO Basel - haben auf dieses Bedürfnis reagiert: Am 28. April 1999 gründeten sie die EQUAM Stiftung zur Förderung der externen, unabhängigen Qualitätsentwicklung in der medizinischen Versorgung. Seit 2000 stehen den Patientinnen und Patienten und den EQUAM-zertifizierten Organisationen

\section{Drei leitende Ärzte haben im April 1999 die EQUAM Stiftung gegründet.}

unabhängige Ombudsärzte zur Schlichtung von Konflikten in medizinischen Belangen zur Verfügung. Ein weiterer wichtiger Meilenstein für die EQUAM Stiftung waren ihre Akkreditierung durch die Schweizerische Akkreditierungsstelle (SAS) als Zertifizierungsstelle für Managementsysteme in der ambulanten

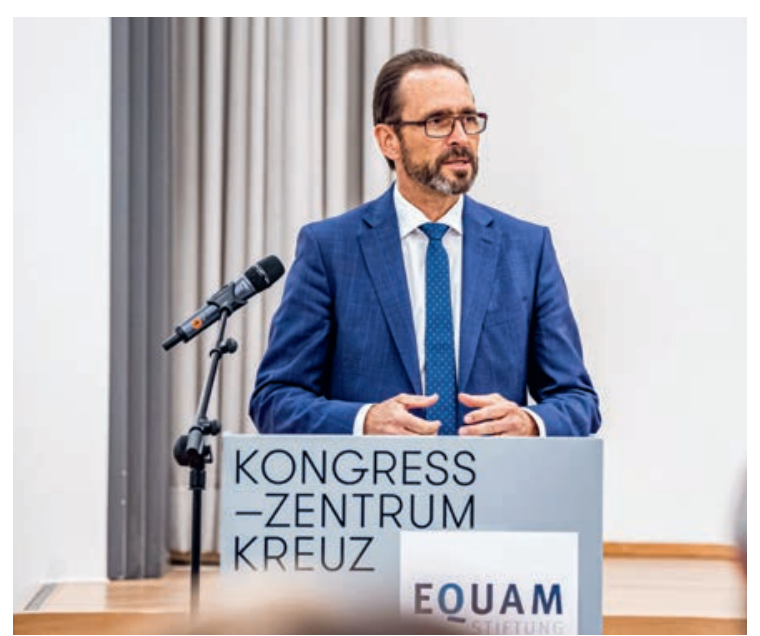

Pascal Strupler, Direktor BAG, ist überzeugt: «Das EQUAMZertifikat kann für die Patientinnen und Patienten bei der Wahl ihres Hausarztes entscheidend sein.»

Medizin im Januar 2003 und die regelmässigen ReZertifizierungen.

\section{Zehn Prozent packen die Qualitätsarbeit freiwillig an}

Pascal Strupler, Direktor Bundesamt für Gesundheit, anerkannte die Arbeit an der Jubiläumsfeier: «Vor 20 Jahren haben die drei Gründer eine Pionierarbeit geleistet. Es ist ermutigend, dass die Anzahl der EQUAM-zertifizierten Ärztinnen und Ärzte steigt. Aktuell arbeiten in der Schweiz rund zehn Prozent der Ärztinnen und Ärzte im ambulanten Sektor mit einem Fachtitel in Allgemeiner und Innerer Medizin in einer 


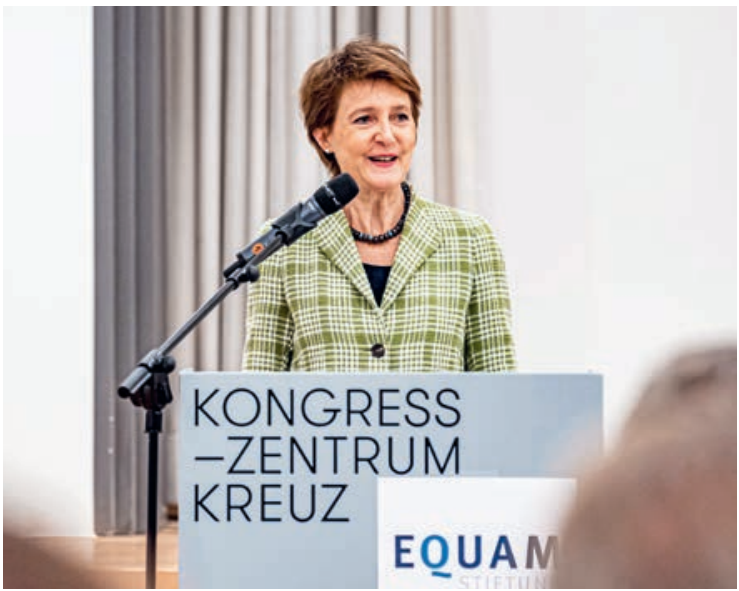

Bundesrätin Simonetta Sommaruga, Stiftungsratspräsidentin EQUAM von 2008 bis 2010, hielt das Grusswort.

EQUAM-zertifizierten Praxis. Bei zehn Prozent von ermutigend zu sprechen ist mutig. Aber diese zehn Prozent packen die Qualitätsarbeit freiwillig an.»

\section{Qualität ist kein Zufall}

Es ist erfreulich, dass sich innovative Ärztinnen und Ärzte, Krankenversicherer und weitere Player aus dem privaten und öffentlichen Gesundheitswesen immer mehr für die Qualität engagieren. Mit der EQUAM Stiftung treiben sie die Qualitätsentwicklung und Patientensicherheit in der ambulanten Medizin voran.

Bundesrätin Simonetta Sommaruga, die zwischen 2008 und 2010 die Stiftungspräsidentin war, war in ihrem Grusswort an der Jubiläumsfeier überzeugt, dass «man die Qualität im Gesundheitswesen nicht allein den Ärztinnen und Ärzten überlassen kann. Es braucht externe unabhängige Kontrollen in diesem Gebiet, wo es um so viel geht - um die Gesundheit. Transparenz, Kosteneffizienz, hohe Patientensicherheit und die Erfüllung von Patientenbedürfnissen sind für mich keine Widersprüche. Es sind die Voraussetzungen für

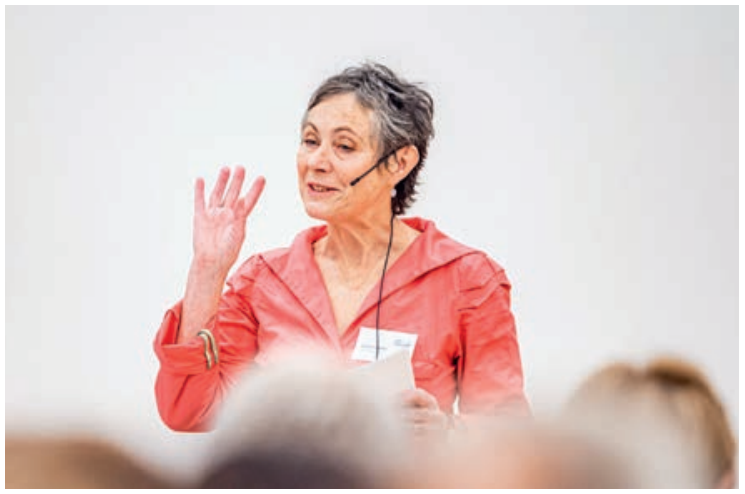

Paula Bezzola, Geschäftsführerin EQUAM, führte rund 70 Gäste durch die Jubiläumsfeier durch. die Qualität. Die Hartnäckigkeit und Unbestechlichkeit der EQUAM Stiftung beeindrucken mich und ich bin froh, dass es EQUAM gibt.»

Mit der KVG-Revision, Artikel 58, und mit der neuen Verordnung zur Integrität und Transparenz im Heilmittelbereich VITH entstehen neue wichtige gesetzliche Grundlagen. Alle Stakeholder und die noch zu bildende Qualitätskommission des Bundes stehen in der Verpflichtung, diese Gesetze und Verordnungen in den nächsten Monaten und Jahren umzusetzen: Indem die EQUAM-Zertifizierungen in den geforderten Verträgen anerkannt werden, kann ein zusätzlicher finanzieller Anreiz für die Arztpraxen geschaffen werden, sich EQUAM-zertifizieren zu lassen.

\section{Qualität im Alltag integrieren}

Der Bund, die Versicherer, die Kantone, die FMH, Fachgesellschaften und weitere Stakeholder haben es jetzt in der Hand, die Qualitätsentwicklung in den Arztpraxen intensiver zu fördern und verbindliche Resultate zu fordern. Es gibt viele engagierte Praxen, die diesen Weg schon einige Jahre zusammen mit der EQUAM Stiftung gegangen sind.

Auch wenn es für einige Ärztinnen und Ärzte eine Herausforderung ist, sich über die Schulter schauen $\mathrm{zu}$ lassen, ist eine externe neutrale Sicht für eine wirkungsvolle Qualitätsarbeit unabdingbar. Die Qualitätsentwicklung ist ein Führungsthema und braucht einen klaren Rahmen: Welche Aspekte der Qualität werden nach welchen Kriterien untersucht? Welche Massnahmen müssen umgesetzt werden, bis wann und wer ist

\section{EQUAM-Zertifizierungen}

Das Zertifizierungsprogramm für Gesundheitseinrichtungen beschäftigt sich mit Struktur-, Prozess- und Ergebnisqualität der Gesundheitsversorgung. Analysiert werden fünf Bereiche: Infrastruktur, Patientinnen und Patienten, Team, Koordination mit Externen und Qualität \& Sicherheit.

Die Programme der Behandlungsqualität beinhalten mehrere Module: Medikationssicherheit sowie die Behandlungsqualität für spezifische Patientengruppen (zurzeit für die Diagnosen Diabetes mellitus, arterielle Hypertonie und koronare Herzkrankheit). Aufgrund der Analyse einer Auswahl von Krankenakten durch einen externen Auditor werden Massnahmen zur Qualitätsoptimierung mit dem behandelnden Arzt identifiziert und umgesetzt.

Beim Programm für Ärztenetze werden unter anderem Fragen der Führung, der Qualitätsarbeit und der Anwendung von Guidelines überprüft und optimiert. Wichtige Voraussetzung für dieses Zertifikat ist, dass mindestens 80 Prozent der angeschlossenen Arztpraxen das Programm für Gesundheitseinrichtungen bestanden haben oder 80 Prozent der hausärztlichen Tätigkeit der jeweiligen Praxis mit einem der Behandlungsqualitätsprogramme zertifiziert sind. 


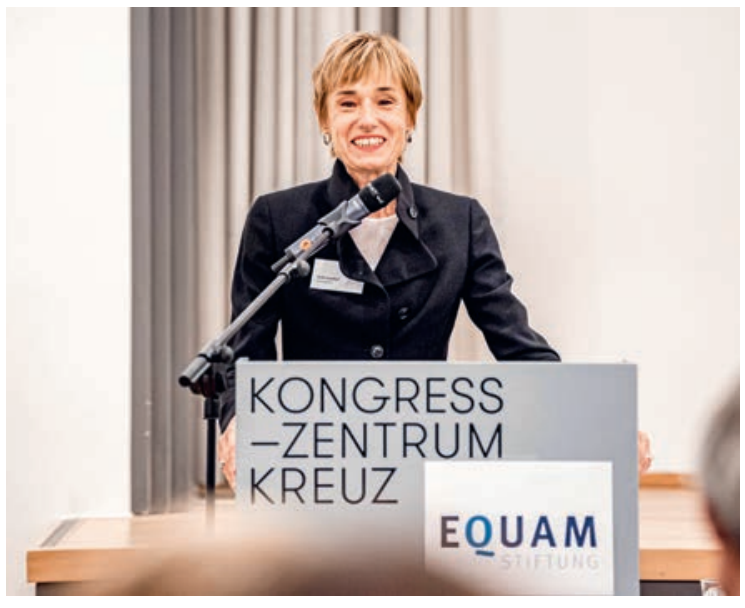

Ruth Humbel, Nationalrätin, Stiftungsratspräsidentin EQUAM, warf Blick auf die aktuelle politische Situation.

dafür verantwortlich? Auf den ersten Blick einfache Fragen, doch der Alltag holt die Praxen schnell ein. Die EQUAM Stiftung führt die Praxen und Ärztinnen und Ärzte durch den Qualitätsprozess nach den SAMWEmpfehlungen durch [1].

Die Gespräche mit den Ärztinnen, Ärzten und MPA im Rahmen der Audits zeigen klar: Die Zertifizierung von Arztpraxen fördert die Entwicklungsdynamik und trägt dazu bei, die Arbeitsbedingungen der Gesundheitsprofis zu verbessern. Ausserdem leistet die Zertifizierung einen Beitrag zur Vereinheitlichung und Dokumentation der Prozesse, was wiederum zur Fehler- und Kostenreduktion führt. Die Zertifizierung allein ist zwar noch keine Garantie für Qualität. Sie erleichtert den Ärztinnen und Ärzten jedoch die Erfüllung von Anforderungen, welche für einwandfreie medizinische Dienstleistungen notwendig sind. Zudem hilft sie den Praxen, eine Kultur der stetigen Qualitätsentwicklung zu verankern.

Es hat sich bewährt, die EQUAM-Indikatoren auf die spezifische Ausgangslage und den klinischen Alltag in einer Arztpraxis in der Schweiz auszurichten. Die EQUAM Stiftung beobachtet eine steigende Nachfrage nach dem Programm zur Medikationssicherheit. Dies hängt mit der hohen Fragmentierung des Gesundheitswesens in der Schweiz zusammen. Viele Schnittstellen erschweren die sichere Medikation. Ein gut informierter Patient und der Hausarzt als seine Anlaufstelle rücken als Drehscheibe in den Vordergrund und brauchen Unterstützung. Diese bieten die Qualitätsprogramme der EQUAM Stiftung.

\section{Auf dem richtigen Kurs}

Die EQUAM-zertifizierten Praxen können positiv in die stärker regulierte Zukunft blicken. Sie wissen bereits, was kontinuierliche Qualitätsentwicklung ist, und erfüllen schon heute hohe Qualitätsanforderungen. Sie sind es sich gewohnt, eine externe Sicht auf ihre Strukturen, Prozesse und Arbeitsweise zu erhalten und sich mit anderen Praxen zu vergleichen. Die Resultate des externen Audits und der Befragung(en) werden mittels eines Benchmarks zurückgespiegelt.

Für die zukünftigen Entwicklungen auf nationaler Ebene ist die EQUAM Stiftung daran interessiert, die Reichweite der Zertifizierungsprogramme auszudehnen. Sie strebt nach Kooperation und Harmonisierung der verschiedenen Bemühungen zur Qualität im Gesundheitswesen. Zusammen mit den Auditorinnen und Auditoren, mit den zertifizierten Praxen und vielen Fachpartnern treibt die EQUAM Stiftung mit Know-how und Leidenschaft die Qualität im Schweizer Gesundheitswesen voran.

\section{Bildnachweis}

(c) susannegoldschmid

\section{Literatur}

1 SAMW, Zertifizierung im medizinischen Kontext (2011), https://www.samw.ch/de/Publikationen/Empfehlungen. html: Download 18.11.2019.

\section{Das Wichtigste in Kürze}

- Ende Oktober feierte die EQUAM Stiftung ihr 20-jähriges Bestehen, namhafte Referenten würdigten die Pionierarbeit der Stiftung für Qualität in der ambulanten Medizin.

- Die Interessenvertreter erwarten, dass die verabschiedete Revision des KVG zur Qualität und wirtschaftlichen Verbesserung, die Verpflichtungen bei der Umsetzung von Qualitätsmassnahmen beinhaltet, die Relevanz ihrer Arbeit weiter erhöhen wird.

- Durch die Anerkennung der EQUAM-Zertifizierung in den erforderlichen Verträgen ist es möglich, einen zusätzlichen finanziellen Anreiz für die von EQUAM zu zertifizierende Arztpraxis zu schaffen.

\section{L'essentiel en bref}

- A la fin octobre, la Fondation EQUAM a fêté ses 20 ans d'existence, des orateurs renommés ont rendu hommage au travail de pionnier de la fondation pour la qualité en médecine ambulatoire.

- Les intervenants s'attendent à ce que la révision adoptée de la LAMal sur le renforcement de la qualité et de l'économicité, qui prévoit des obligations dans la mise en œuvre de mesures de qualité, accentue encore davantage la pertinence de son travail.

- En reconnaissant la certification EQUAM dans les contrats nécessaires, il est possible de créer une motivation financière supplémentaire pour que les cabinets médicaux se fassent certifier par EQUAM. 